\title{
Improving Knowledge for Teaching Mathematical Argumentation in Primary Classrooms
}

\author{
Pi-Jen Lin \\ National Tsing Hua University, Taiwan, ROC
}

\begin{abstract}
This study examined the effects of improving teachers' knowledge for teaching argumentation in primary classroom settings. Six teachers learned pedagogical knowledge for facilitating students' argumentation in six classrooms and a teachers learning community. The data collection included video- and audio-tapes of classroom observations and weekly meetings, lesson plans, and students' worksheets. The pedagogical knowledge the teachers learned for facilitating students' argumentation in classrooms resulted in: (1) Learning meanings and the ways of formulating conjecture; (2), Identifying norms for sharing conjectures between small groups; (3) Learning four aspects for checking conjectures; (5) Distinguishing differences between incorrect and false conjectures, validating and categorizing conjectures, and validating and justifying conjectures.
\end{abstract}

Keywords: Argumentation, classroom discourse, formulating conjectures, validating conjectures.

The importance of engaging students in classrooms activities including conjecturing, generalizing, and justifying has been increasingly emphasized in international mathematics education reforms. For instance, the Standards of Mathematics Practices (Common Core State Standards Initiative, 2010) call for students to construct viable arguments and critique the reasons of others. Students should have the opportunity to make conjectures, communicate their conjectures to others, explore the truth of their conjectures, use counterexamples to refute a false conjecture, respond to the arguments of others, and justify their conclusions. However, the significant shift away from traditional instructional models, in both what and how mathematics is taught is a challenge for classroom teachers (National Mathematics Advisory Panel, 2008). Kuhn (1991) highlights the use of valid argumentation, but also acknowledges that it does not come naturally and is acquired only through practice. This indicates that argumentation needs to be explicitly taught, through appropriate activities, support, and modelling.

Several researchers agree that teacher plays a pivotal role in orchestrating mathematical discourse (e.g., Staples, 2007; Yackel, 2002). Teachers need to know more about argumentation and also need to acquire the 
pedagogical skills to promote classroom practices, such as prompting students to establish claims and justifications; encouraging students to critically consider different arguments; presenting to students what constitutes acceptable mathematical arguments; and modeling particular ways of constructing arguments. Given the challenges for shifting away from traditional approaches to effective engage students in mathematical discourse, it is necessary to have learning communities similar to lesson study (Fernander \& Yoshida, 2004) for supporting the teaching of argumentation in mathematics classrooms. Teachers also need to be supported in planning carefully and incorporating conjecturing into their argumentation objectives.

Hence, this study was intended to identify what teachers needed to know for learning how to teach argumentation in primary classrooms. The research question explored: What are the aspects of pedagogical knowledge needed for formulating conjectures and validating conjectures, as part of the processes of argumentation?

\section{Conceptual Framework}

\section{Teachers' Learning Communities}

Vescio, Ross, and Adams (2008) indicate positive impacts result for teachers in learning communities on practice and student learning. However, there is not sufficient time for teachers to work together in such communities. To make substantial instructional changes that impact student learning, there is a need for teachers to have the opportunity to learn through collaborative discussion that focuses on student learning (Stoll, Bolam, McMahon, Wallace, Thomas, 2006). For instance, protocols can be used by students, in lesson plans or in learning tasks to improve student learning. Little and Curry (2009) suggest that protocol-based conversations have the potential to elicit broad participation and to build communities. However, protocols alone are insufficient to ensure teacher learning. Thus, setting up a teacher learning community for investigating instruction through collaboratively designed tasks that are implemented in observable lessons is essential. In this study, such tasks are considered as a protocol-based tool for helping teachers to learn to teach argumentation. Observable classroom lessons were also included to provide a context for the teachers to recognize and identify the pedagogical strategies necessary for promoting argumentation skills (Fernander \& Yoshida, 2004).

\section{Conjecturing for Evoking Argumentation in Classrooms}

Argumentation is highly related to conjecturing. According to Simon, Erduran, and Osborne (2006), argumentation is the process of assembling the components of the substance of claims, data, warrants, and backings contributing to the content of the argument. To facilitate mathematical argumentation, teachers must actively help students build an appropriate 
understanding of mathematics concepts. According to Stein, Engle, Smith, and Hughes (2008), this process begins with choosing or designing high cognitive demand tasks. Lin and Tsai (2016) suggest that conjecturing is a pedagogy for evoking students' argumentation. In particular, for elementary students, a conjecture can be made based on the observation of a finite number of discrete cases, in which a consistent pattern is observed. Cañadas and Castro (2005) offer seven stages for conjecturing based on finite discrete cases: (1) observing cases, (2) organizing cases, (3) searching for and predicting patterns, (4) formulating a conjecture, (5) validating the conjecture, (6) generalizing the conjecture, and (7) justifying the generalization. The stages of conjecturing are considered for this study as the basis for supporting teachers in designing the tasks for primary students engaging in argumentation activities. The meaning of argumentation in the study is adopted from Simon, Erduran, and Osborne's study (2006) as the process of assembling the components of the substance of claims, data, warrants, and backings contributing to the content of the argument.

\section{Facilitating Mathematics Argumentation}

Excepting the importance of the tasks, teachers need to actively engage in guiding whole-class discussions toward worthwhile mathematical argumentation. However, a challenge to face for those who attempt to use conjecturing-based approach of instruction is that solution paths are usually not anticipated when planning the tasks (Stein et al., 2008). Stein et al. (2008) propose a five practices model for orchestrating whole-class discussion that are conducted after students respond to the high-cognitively demanding tasks. The five practices are: (1) anticipating students' responses to the tasks, (2) monitoring students' response to the tasks, (3) selecting particular students to present their mathematics responses, (4) purposefully sequencing the students' responses that will be displayed, and (5) making connections between different students' responses.

In addition, research indicates that the questions a teacher asks influence the nature of a classroom discussion (Hufferd-Ackles, Fuson, \& Sherin, 2004, 2015; Staples, 2007). Hufferd-Ackles et al. (2015) suggest that teachers should create a "math-talk learning community" in a classroom for facilitating productive discourse. They identify four components for a mathtalk community including teacher role, questioning, explaining mathematical thinking, mathematical representations, and building student responsibility within the community.

For primary school students with limited mathematics knowledge and experience of argumentation in classroom discourse, the uncertain conjectures arriving at a conclusion is challenging without a teacher's guidance. Stein et al.'s (2008) five practices model for orchestrating whole-class discussion were adapted in this study for organizing students' worksheets, and Hufferd-Ackles et al.'s (2015) "math-talk learning community" was adapted in the teacher 
questioning.

\section{Research Method}

\section{Participants}

This multiple-case study included six teachers who willing to initiate classroom changes. Two were fourth grade teachers (T1, T2), two were fifth grade teachers (T3, T4), and two were sixth grade teachers (T5, T6). T1, T3, and $\mathrm{T} 5$ teachers never taught conjecturing, while T2, T4, and T6 had one year of experience with conjecturing as an instructional approach for a year. The size of each class ranged from 18-27 students.

\section{A Learning Community of Teachers}

The study included data from six classrooms and a teacher learning community with weekly routine meetings. The learning community included six teachers and the researcher in a three-year project known as the Teacher Professional Development of Designed-Based Conjecturing for Argumentation (TDCA). The weekly meetings were focused on theoretical perspectives for teaching argumentation and according to the needs of the teachers as the study progressed.

The teacher and the researcher met to collaboratively plan lessons from analyzing the instructional textbook, conduct pre-tests, write lesson plans, observe lessons, and to reflect on the observed lessons. The researcher provided both theoretical ideas and practical resources to stimulate change in teachers' practices, and the teachers shared their practices with the researcher to inform her thinking. The weekly meetings also provided opportunities for the teachers to listen to different perspectives, to reshape their own views, and to plan next steps for improving instruction and student work. The aim of the learning community was to facilitate teacher development through encouraging teachers to take ownership by designing their own conjecturing tasks and carrying out the tasks. The tasks were designed with the ADDIER model with an acronym meaning: Analyzing textbooks, Developing the potential tasks, Designing the draft tasks, Implementing, Evaluating and Revising the draft for the following day or another classrooms. Each task was designed according to the five stages of conjecturing. Each stage was explored individually, in groups, and with the whole class when possible. The ADDIER model and the five stages of conjecturing approach as part of the research design of the study are displayed in Figure 1. The modification of the seven stages reduced into five stages for designing the tasks for conjecturing is not the focus of the paper, but it was described in a previous one authored by Lin and Tsai (2016). 


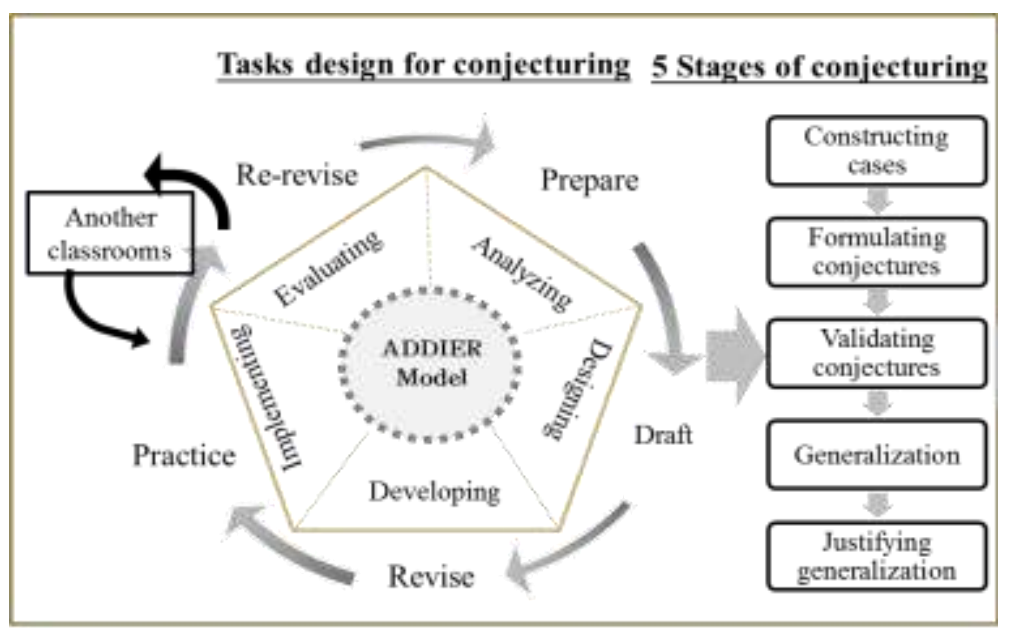

Figure 1. A model of task design for conjecturing: ADDIER model.

Each teacher was asked to incorporate at least two conjecturing-based lessons for promoting students' argumentation across the mathematics topics in a year.

\section{A Typical Lesson for Teaching Argumentation}

A typical teaching model for argumentation often proceeds in five stages of conjecturing (Lin \& Tsai, 2016). It begins with the "constructing cases" stage. During this stage, students are expected to engage in construing cases, compiling, organizing, and observing the individual cases, and looking for patterns. The cases to be constructed became the data for making a conjecture as the next stage of teaching argumentation. The second stage includes "formulating conjectures" in which students make conjectures based on only the cases they constructed in the first stage. The question "What can you discover?" was used for formulating the conjectures. This stage often started with individual work and then was followed by small groups and whole class discussions. At this stage, students were encouraged to make conjectures and were prepared to explain their conjectures to others in a small group and whole class discussion. The conjectures based on observing several cases sometimes led students to incorrect conjectures or claims. The question such as "share your conjectures and mutually examine in a group." was used to further check the correctness of students' conjectures. The conjectures led to the conclusion, as a component of the argumentation. "Validating conjectures" was the third stage for validating the conjectures as true for new cases. The question "How do you validate your conjectures?" was used for students to validate their own conjectures. The fourth stage "generalizing the conjectures" generalized the conjectures to all the cases. The universal quantifiers "for all" or qualifier "as long as", "under the condition of ..." were involved in this stage for the conjecture to be true for all cases. "Justifying the generalization" was the final stage of the teaching model for argumentation. 
The deductive reasoning stage was used for justifying the truth of the conjectures. The lesson frequently concluded with a whole-class discussion and summary of various student-generated conclusions. Part of the conclusions were also lesson objectives stated in the textbooks.

Students in each of the six classrooms were organized heterogeneously in groups of 4 or 5 students. Students worked individually or with their small groups for solving and recording solutions on B3 paper. Within the classes, classroom discourses were expected to reflect students' conjecturing, explanation, and justification for how a task was examined.

\section{Data Collection and Data Analysis}

Data collection occurred during the first year of the TDCA project and included 12 tasks for conjectures, students' worksheets from each class, 12 video-tapes of classroom observations, and 12 weekly meetings of the learning community. The video recordings of each lesson and weekly meetings were transcribed. The transcripts were used as primary data sources, supplemented by the students' worksheets. The data analysis of the transcripts focused on the teachers' oral contributions for facilitating students' argumentation. If an utterance was intended to achieve argumentation, then it was coded. Since each lesson had different discourse structures, we did not focus on the frequencies of the codes, instead, the pedagogical strategies for promoting students' argumentation became the focus of the discourse analysis. A coding scheme was derived from a grounded approach that focused on the processes needed to facilitate argumentation. The coding scheme was refined and extended through contrasting and comparing codes. The teachers' role in questioning, pedagogy, and knowledge required for facilitating students' argumentation were identified drawing upon the five stages of conjecturing. However, only formulating conjectures and validating conjectures in the second and the third stages of conjecturing approach were reported in this paper.

The codes were reviewed and analyzed by two graduate students and the researcher. Inter-rater reliability was established with a mutual agreement rate of $95 \%$ among the coders.

\section{Results}

\section{Formulating Conjectures Stage}

After the cases being constructed, each teacher assisted students to formulate conjectures based on the cases. The teachers' knowledge and pedagogy of teaching argumentation at the conjecture stage included the meaning of formulating conjectures, the four aspects of checking conjectures, the distinction of incorrect conjectures from false conjectures, the norm of sharing conjectures within a small group, and compiling individualconjectures into group-conjectures 
Learn to know the meaning of formulating conjectures. In the second stage of conjecturing, each teacher started with asking individual students to make conjectures within a small group. The stage was not only for producing various conjectures around a task but also for encouraging individual participation. Teachers made attempts to help students to know what the formulation of conjectures means. The $\mathrm{T} 1$ teacher with little experience of conjecturing started to ask students by "what do you discover aligned with the cases you constructed?". She was not satisfied with her students' superficial numeral relationships without relevant knowledge of mathematics properties. In the weekly meeting, the T2 teacher suggested to the T1 teacher to question by asking "what mathematical ideas or mathematical relations do you discover aligned with the cases you constructed?". From then on, the 20-25 students in each class formulated 2030 conjectures, but many of them could not arrive at the objectives of the lesson. Instead, most of the conjectures had similar mathematics ideas they had previously learned. At the end of the yearly study, the question was a prompt for helping students to formulate conjectures created by the teachers, by asking "“what naive or creative mathematical ideas or mathematical relations do you discover aligned with the cases you constructed?"

Learn to know the norms for sharing conjectures within a small group. After formulating individual conjectures, it was immediately followed by group sharing which was more likely to make the argumentation taking place in classrooms. Students were encouraged to work in groups, listen carefully, and articulate clearly their own ideas. For the three teachers, T1, T3, and T5, they learned the skill of articulating group sharing from T6's lesson. After the individual conjectures, each teacher asked students to mutually check the conjectures in their small group. The following excerpt is taken from T5's lesson where she helped students to establish a norm for sharing individual conjectures.

T5: ..... When working in groups, you need to share your own ideas, listen cautiously, and ask questions for others. Reporter needs to speak loudly and slowly. Listeners need to pay attention to listen what the speaker said and also need to ask a question to the speaker.

T5 : When do you ask a question?

All : As the speaker finishes the report.

T5: What jobs are the $\# 1$ and $\# 4$ or $\# 5$ students in each group?

All: Assisting in Holding the reporter's worksheet to present all information to be seen for each member of the group. 
T5: How many minutes you need to be done for this sharing in a group? (be continued)...

Learn to know the four aspects for checking conjectures. To help students to have mathematical literacy, they need to learn to be a person whose speech must rely on reasonable evidence. It was not acceptable for teachers to think that even though a conjecture was true, it should be considered a case in this study. For instance, the students in the T5 class made a true conjecture "diameter $\times 3.14=$ perimeter of a circle" as in Figure $2(\mathrm{~b})$, but the number 3.14 was not presented in the worksheet, as shown in Figure 2 (a). Thus, this conjecture was not accepted, even though it was a true statement. It is called as non-data conjecture. The meaning of checking a conjecture was addressed in a weekly routine meeting. The first aspect of checking conjectures was that the conjecture should be based on cases.

Task 1: Ratio of perimeter to diameter of circle

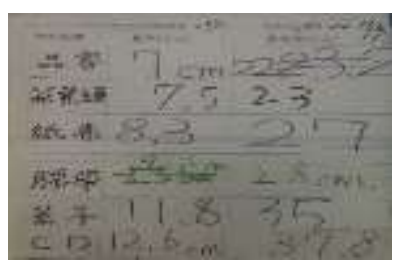

(a) Cases constructed by group 5

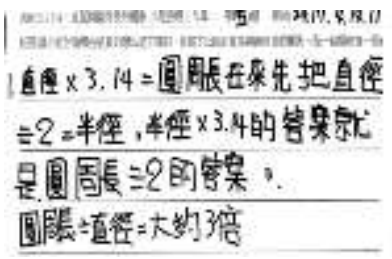

(b) Chinese version diameter $3.14=$ perimeter of a circle.

Then diameter $\div 2=$ radius, Radius $\times 3.14=$ perimeter $\div 2$

Figure 2. An incorrect conjecture formulated by group 5.

The second aspect of checking conjectures was the conjecture was rationally incorrect, even though it is based on the construed cases. For instance, one incorrect conjecture generated by S14 in group 3 of T5's triangle lesson was "if the two shortest sides are greater than the third side, then it is not a triangle." As observed, it was common among the six classrooms in the first two months of the study that the majority of the students within a small group only focused on listening, but did not identify which of the conjectures were correct.

The third and four aspects of checking conjecture were acceptable tentatively, since those conjectures were not only correct, but also counted on cases. The non-true conjecture was true for some of the cases, but not true for all cases. Thus, a non-true conjecture is not true for all cases, in the third aspect of checking conjectures. The fourth aspect of checking conjectures involved the conjecture was always true for all cases. It is called as true conjecture. The four aspects of checking a conjecture are summarized in Table 1. 
Table 1

Four Aspects of Checking a Conjecture

\begin{tabular}{cccc}
\hline $\begin{array}{c}\text { Aspects of } \\
\text { conjecture }\end{array}$ & Rely on cases & Tentative accept & Always true \\
\hline Non-data & $\mathbf{x}$ & $\mathbf{x}$ & -- \\
Incorrect & $\mathrm{o}$ & $\mathbf{x}$ & -- \\
None-true & 0 & 0 & $\mathbf{x}$ \\
True & 0 & 0 & 0 \\
\hline
\end{tabular}

Learn to distinguish incorrect conjectures from false conjectures. It is essential to distinguish between incorrect and non-data conjectures before validating conjectures. The incorrect conjecture could be neither be rationally correct nor be generated from the cases on the worksheet.

At the beginning of the study, the teachers were confused with the distinction between incorrect conjectures from non-true conjectures. For instance, in a multiplication task of decimals, T6 invited students to refute S20's non-ture conjecture from group 3: "If the digit number in ones of the multiplicand is 1, then the digit number in ones of the product must be the same as that of the multiplier." seen in Figure 2. The conjecture was based on the cases, but it was a non-true conjecture, shown in Figure 2(a).

Task 2: The relations between multiplicand, multiplier, and product of decimals.

Group 3 :

(1) $4.1 \times 2.3=9.43$

(2) $4.1 \times 0.6=2.46$

(3) $4.1 \times 1=4.1$

(4) $4.1 \times 1.4=5.74$

(5) $4.1 \times 2.7=11.07$
If the one's digit of the multiplicand is 1 , then the one's digit of the product must be the same as that of the multiplier.

(a) Cases from group 3

(b) S20's correct conjecture

Figure 2. A correct conjecture formulated by S20 in group 3 in T6's class.

Compiling individual-conjectures into group-conjectures. Taking individual conjectures together, about ten conjectures came up within a small group. Within the conjectures, some had similar ideas, but with different statements. It was extremely time-consuming work for formulating groupconjectures from individual-conjectures because students were simply copying and writing on the group worksheets from individual work. This challenge was discussed in a weekly meeting. In the end of the first month of the study, the researcher suggested the teachers reduce the number of groupconjectures into less than 4 selected from ten conjectures in a group. However, this rule of group-conjectures was highly dependent on the more abled students in a group. Consequently, the participation of the low-achievers from 
each group was eliminated. Attempts to accurately compile individual conjectures into group conjectures was unsuccessful because it totally violated the spirit of the conjecturing approach as inspiring the variety of conjectures and the participation of each student in the groups.

This issue was raised again in a weekly meeting. Several strategies were suggested by the six teachers and tried out in their classrooms. For instance, in T4's lesson, he attempted to invite naïve and creative conjectures as group-conjectures in group work. He figured out a strategy that each individual conjecture accepted in a group was written on a strip of paper as a group-conjecture for a whole-class discussion. Another strategy used in T3's lesson, was a slogan positioned at the corner of the blackboard in her classroom. The slogan for compiling individual-conjectures into groupconjectures was: "checking the conjectures agreed in your group first" and followed by "selecting the naïve mathematical ideas agreed in your group".

\section{Validating Conjectures Stage}

Checking the incorrect or false conjectures was found to be effectively completed in small group work with the teacher's guidance. The knowledge of argumentation the teachers acquired at this stage of validating conjectures consisted of: the meaning of validating conjectures, distinguishing validating conjectures from categorizing conjectures, and distinguishing validating conjectures from justifying conjectures.

Learn to know the meaning of validating conjectures. In the midway of the first semester of the study, the six teachers were hindered at the stage of validating group-conjectures which were proposed by each group. They did not clarify the distinction of validating conjectures from checking conjectures. T5 reflected on her teaching in a triangle lesson and reported in a routine meeting as follows.

In fact, I had a tension to deal with so many conjectures coming up from students after I invited the conjectures from each group to be posted on the blackboard. I just copied and followed the teacher's [T6] action. I began by discussing the first conjecture posted on the very right side of the blackboard. I only paid attention to organizing the group-conjures instead of checking and validating the group-conjectures before I moved on to discuss the conjectures from another group. I put the conjectures with the same mathematics ideas into different piles (Meeting, Nov. 13).

Taking the advantage of this opportunity, the researcher helped the teachers to clarify the meaning of the validation conjectures in contrast to checking conjectures. The correctness of a conjecture was through checking if it was based on the cases constructed, while the truth of a conjecture is through the validation of new cases. 
Distinguishing the validation of conjectures from categorizing conjectures. At the beginning, the six teachers struggled with the various group-conjectures. The group-conjectures were sorted by the teachers themselves into different categories based on the same mathematical ideas. The teachers forgot to validate the group-conjecture and to check if similar conjectures were correct. T1 mixed up validating conjectures and categorizing conjectures. For instance, T1's lesson of recognizing triangles, validating a conjecture is the goal of the classroom discourse in the following excerpt, but it was mistakenly acted as categorizing conjectures.

T1: The conjecture from group 2 was that each angle of an equilateral triangle is $60^{\circ}$. Which of you have the similar conjecture?

S3 : Yes, in our group 3 we had the same conjecture with group 2 as each angle of an equilateral triangle is congruent. (T1's teaching)

The appropriate pedagogical strategy of validating a conjecture was addressed in several routine weekly meetings. The researcher helped the teachers to distinguish the validation of a conjecture from organizing the conjectures. Validating a conjecture meant to validate a conjecture with more new cases, while categorizing the conjectures meant to organize the various conjectures into different categories.

Learn to distinguish between validating conjectures and justifying conjectures. The three teachers $\mathrm{T} 2, \mathrm{~T} 4$, and $\mathrm{T} 6$ brought their prior teaching experiences on student-centered approaches into their classrooms. For student-centered approaches, the explanation of each strategy for resolving a given problem was required in classroom discourse. Thus, asking a question included, "Why is it ? Explain it." Conversely, the teachers who relied on the conjecturing approach led to less effective instruction if they asked such questions. Two reasons might explain this situation. First, the students with weak mathematical knowledge were incapable of justifying the truth of a conjecture in this stage. Second, the focus on validating a conjecture was affected by the long process students took with their explanations in each group-conjecture. The explanation or verification of a conjecture was required in this final stage of "justifying conjectures". A final result indicated the teachers appreciated validating conjectures without justifying conjectures because the conjectures were validated by new cases. This approach was perceived as much easier because it appeared to save time with teaching conjectures.

\section{Conclusion and Discussion}

The findings from this study indicate the weekly meetings gave rise to a set of tasks for conjecturing and utilizing pedagogical strategies that can be used in structured norms to facilitate argumentation in classroom instruction. The tasks for conjecturing with five stages played a key role in initiating and 
sustaining the argumentation. Additionally, working collaboratively with teachers led to an initial change in teaching argumentation. This finding led to the conclusion that it is possible for teachers to adapt and develop their teaching for argumentation and to bring about a change in the nature of mathematical classroom discourse. The results from the study also suggest supported Hufferd-Ackles, Fuson, and Sherin's (2015) findings about the creation of "math-talk learning community" in classrooms. Another important result indicated students who engaged in classroom discourse to validate and to justify warrants and data for and against conjectures provided other discourse elements, such as explaining ideas or thinking.

The teachers' initial approach to teach argumentation was not fundamentally altered, but rather, refined and extended over the year. Some of the teachers demonstrated good classroom practices in teaching argumentation, while other teachers appeared not to have a full appreciation of the potential of the alternative instructional approach. Appreciation appeared to depend on the teachers' exiting knowledge, understanding of argumentation, and if student-centered approaches were implemented. Hence, to support teachers making progress in teaching argumentation, the study suggests professional development should be on teachers' existing understanding of teaching argumentation. To this end, this study helps to identify the pedagogical knowledge of argumentation, including the meaning of formulating and validating conjectures, the norm of sharing conjectures within a small group, the four aspects of checking conjectures, the distinction of incorrect from false conjectures, the distinction of validating from categorizing and from justifying conjectures.

This study also helped the teachers to transform pedagogical knowledge of argumentation into classroom discourse. The teachers learning to teach argumentation supporting Stoll's (2006) claims for the need of collaborative discussion focusing on student learning. For instance, before checking conjectures, the teachers learned that students needed to learn how to listen carefully and talk clearly. Before formulating and validating groupconjectures, teachers learned that students needed to learn how to provide the evidence and identify reasons in group discussions. The results implicitly suggest to teacher educators and researchers that pedagogical knowledge of argumentation is a preliminary step for engaging teachers in using argumentation as an instructional approach.

The study supported Vescio, Ross, and Adams's (2008) emphasis of the quality of the teacher learning community on teachers' practices. The routine meetings initiated the process of reflection for action and reflection on action. In the learning community meetings, reflection for action suggested incorporating conjecturing into textbooks and designing learning tasks that promote classroom discourse were important for improving instruction. Moreover, designing and implementing tasks with protocol-based tools might also improve the teaching of argumentation prior to implementation in the 
classroom. Reflection on action also emerged after teaching suggesting the relevancy of examining the implementation of the tasks, analyzing students' worksheets, analyzing teachers' pedagogical strategies, and teachers' oral communication in their classrooms. This study contributed to investigating the classroom as a viable setting for teachers learning how to teach argumentation beyond student-centered lesson study approaches (Fernander \& Yoshida, 2004).

\section{ACKNOWLEDGEMENT}

The research was funded by the Ministry of Science and Technology (MOST 104-2511-S-134 -003 -MY3). Any opinions, findings, conclusions, or recommendations expressed in this paper are those of the author and do not necessarily reflect the views of the MOST. I wish to thank the teachers and students who participated in this research.

\section{References}

Fernandez, C., \& Yoshida, M. (2004). Lesson study: A case of a Japanese approach to improving instruction through school-based teacher development. Mahwah, NJ: Lawrence Erlbaum Associates.

Hufferd-Ackles, K., Fuson, K., \& Sherin, M. G. (2004). Describing levels and components of a math-talk learning community. Journal for Research in Mathematics Education, 35, 81-116.

Hufferd-Ackles, K., Fuson, K., \& Sherin, M. G. (2015). Describing levels and components of a math-talk learning community. In E. A. Silver \& P. A. Kenny (Eds.), More lessons learned from research: Volume 1: Useful and usable research related to core mathematical practices (pp. 125-134), Reston, VA: NCTM.

Kuhn, D. (1991). The skills of argument. Cambridge University Press.

Little, J.W., \& Curry, M.W. (2009). Structuring talk about teaching and learning: The use of evidence in protocol-based conversation. In L.M. Earl \& H. Timperley (Eds.), Professional learning conversations: Challenges in using evidence for improvement (pp. 29-42). Netherlands: Springer.

National Mathematics Advisory Panel (2008). The final report of the National Mathematics Advisory Panel. Washington, DC: U.S. Department of Education.

Simon, S., Erduran, S., \& Osborne, J. (2006). Learning to teach argumentation research and development in the science classroom. International Journal of Science Education, 28(2-3), 235-260.

Staples, M. (2007). Supporting whole-class collaborative inquiry in a secondary mathematics classroom. Cognition and Instruction, 25(2-3), 161-217. 
Stein, M. K., Engle, R. A., Smith, M. S., \& Hughes, E. K. (2008). Orchestrating productive mathematical discussions: Five practices for helping teachers move beyond show and tell. Mathematical Thinking and Learning, 10, 313-340.

Stoll, L., Bolam, R., McMahon, A., Wallace, M., \& Thomas, S. (2006). Professional learning communities: A review of the literature. Journal of educational change, 7(4), 221-258.

Lin, P. J. \& Tsai, W. H. (2016). Enhancing students' mathematical conjecturing and justification in third-grade classrooms: The sum of even (odd) numbers. Journal of Mathematics Education, 9(1), 1-15.

Vescio, V., Ross, D., \& Adams, A. (2008). A review of research on the impact of professional learning communities on teaching practice and student learning. Teaching and Teacher education, 24(1), 80-91.

Yackel, E. (2002). What we can learn from analyzing the teacher's role in collective argumentation? Journal of Mathematical Behavior, 21, 423440 .

\section{Author:}

Pi-Jen Lin

National Tsing Hua University, Taiwan, ROC

E-mail:linpj@mx.nthu.edu.tw 